
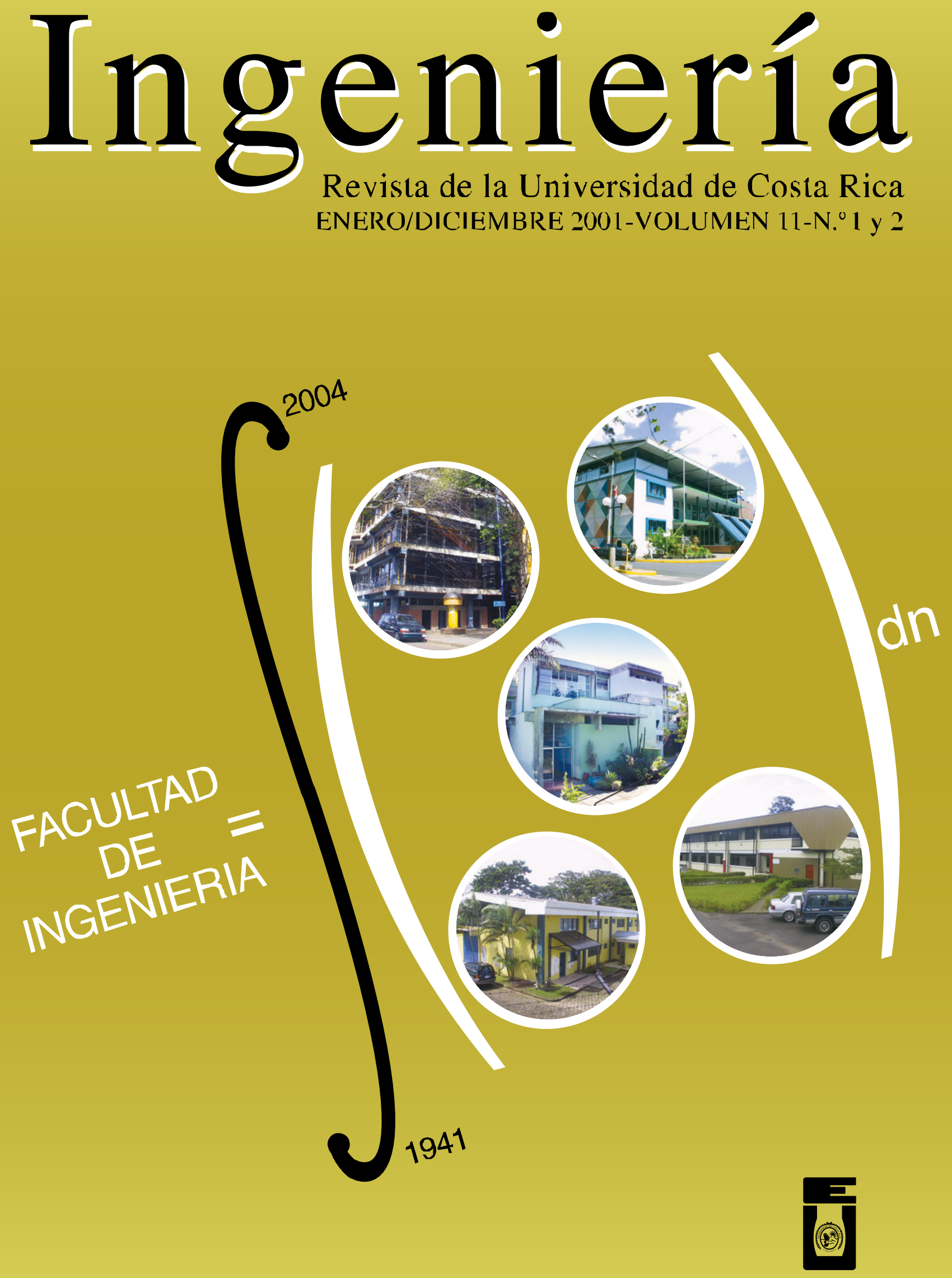


\title{
LA NECESIDAD DE VALORAR LOS EQUIPOS DIDÁCTICOS
}

Ing. Luis Arturo Rapso*

\begin{abstract}
Resumen
Este documento analiza el problema de la selección adecuada del equipo de laboratorio y del material didáctico para la enseñanza de la Ingeniería. A partir de algunos conceptos teóricos se establece un grupo de requisitos que los equipos deben cumplir para este fin.

El objetivo del artículo es formular las bases para la discusión del tema, considerado de fundamental interés por los profesores de Ingeniería.
\end{abstract}

\begin{abstract}
This paper analyses the problem of choosing an adequate laboratory equipment or didactical material for engineering learning. Using some theoretical concepts to develop knowledge a requirement list is established for didactical and laboratory equipment. The objective of this paper is to put in discussion this theme, it is considered fundamental for teachers in engineering.
\end{abstract}

\section{INTRODUCCIÓN}

Uno de los retos al que se enfrenta la enseñanza de la Ingeniería es el de la utilización de material didáctico que cumpla adecuadamente con la función pedagógica; es decir, que sirva como cata-lizador en el proceso de aprendizaje. Paralelamente, existen algunos problemas que deben ser superados previamente.

El primer problema que debe ser solventado está vinculado al ámbito económico. Existen diversos fabricantes en Norte América, Europa y Asia que ofrecen una gran variedad de equipos de buena calidad; sin embargo, su adquisición es bastante onerosa para las universidades públicas del tercer mundo.

La segunda interrogante guarda relación con esto último, pues tratándose de elevadas inversiones se hace necesario establecer criterios que permitan evaluar la efectividad didáctica de los equipos, que llamaremos en adelante "objetos", dada la variedad de formas y características que pueden presentar.
En vista de todo lo anterior, la problemática referida nos sirve de justificación para la rea-lización del presente artículo, en el cual se intentarán establecer algunos lineamientos y criterios que permitan valorar el equipo didáctico. Creemos que parte del éxito de este trabajo se reflejará en futuros esfuerzos que permitan desarrollar materiales didácticos dentro de las propias escuelas de Ingeniería.

\section{REVISIÓN TEÓRICA}

\subsection{PROCESO DE APRENDIZAJE:}

Aunque este trabajo no pretende ser exhaustivo, su objetivo principal consiste en caracterizar un modelo compatible con la realidad de los profesores y estudiantes de una escuela de Ingeniería.

El proceso de aprendizaje, especialmente en las distintas disciplinas que comprende la Ingeniería, suele desarrollar en el estudiante la capacidad para enfrentarse a diversos problemas. 
Existen varias tendencias en el enfoque de la enseñanza de un curso de Ingeniería, a continuación serán citadas las más representativas:

\subsubsection{CONDUCTIVISMO:}

El Conductivismo introduce una visión objetiva de la sociedad y concibe el aprendizaje como una modificación de la conducta. El resultado es una continua repetición de situaciones idénticas. Esta concepción deja de lado la individualidad del estudiante, así como el efecto del entorno social. El método seguido por esta corriente sistematiza la enseñanza, fragmentando los contenidos en elementos menores, lo que no garantiza que se alcance un conocimiento integrado.

\subsubsection{APRENDIZAJE POR DESCUBRIMIENTO:}

Esta teoría, atribuida al psicólogo norteamericano Bruner [1], posee una visión más global del estudiante, en la medida en que el proceso de aprendizaje del individuo está correlacionado con su entorno social y psicológico. Los lineamientos propuestos por esta teoría buscan dar libertad al estudiante para que desarrolle su propio esquema de aplicación de conocimientos en la solución de problemas. Básicamente, lo que procede es la organización de un conjunto de conocimientos fundamentales y de gran injerencia en el tema desarrollado.

Por ejemplo, si se plantea una adecuada exposición de la primera ley de Newton junto con algunas nociones de metodología racional para resolver problemas de estática, el estudiante tendrá las herramientas para resolver problemas complejos, así como una base firme para introducirse en el concepto de la segunda ley de Newton y visualizarla como una generalización que incluye a la primera.

\subsubsection{APRENDIZAJE SIGNIFICATIVO:}

En esta teoría los contenidos están organizados de manera jerárquica. Se parte de conceptos que tienen un mayor poder explicativo. A partir de esa base, se desarrollan otros conceptos de forma constructiva. Estos encadenamientos son conocidos como "mapas conceptuales".

Generalmente, los docentes utilizan algunas de las teorías expuestas o una mezcla de todas ellas. Esto se explica debido a que en muchos casos, los profesores de Ingeniería, así como de otras disciplinas, no se preocupan por realizar estudios formales en el campo pedagógico; por el contrario, sus clases se suelen fundamentar en la experiencia personal como estudiantes. A partir de esta experiencia, los docentes se comportan en el aula como algunos de sus ex-profesores, a los que consideran "buenos" los imitan y a los "malos" ...

Volviendo a las teorías de aprendizaje, según nuestro criterio, es conveniente en primer lugar, resaltar algunos aspectos del aprendizaje por descubrimiento, pues introduce unas condiciones más estimulantes para el estudiante y al mismo tiempo coloca el binomio profesor/estudiante ante un mayor reto, debido a que los conocimientos se desarrollan de una manera más formativa y acorde con los aspectos del entorno.

En segundo lugar, el aprendizaje significativo, gracias a la estructura de mapas conceptuales, integra el proceso de aprendizaje en sus diferentes ámbitos, tanto en el de la transferenciageneración de conocimientos, como en el de la creación de los currículum, metodologías y evaluaciones.

\subsection{LA NECESIDAD DE PLANEAMIENTO DENTRO DE LOS PROCESOS DE APRENDIZAJE:}

Como en cualquier otra actividad humana, es clara la necesidad de un planeamiento de la enseñanza. El Profesor Fernando Murillo[2] plantea que la ausencia de planificación genera rutina, improvisación, pérdida de tiempo y de recursos. 
Los principales aspectos que deben ser considerados en los procesos de planificación son:

¿Qué y para qué se va a enseñar?

¿Cómo se va a enseñar?

¿A quién se va enseñar?

\subsection{LA COMUNICACIÓN DENTRO DEL PROCESO DE APRENDIZAJE}

Es prácticamente imposible obviar el hecho de que el proceso de aprendizaje se desarrolla dentro del marco de la comunicación. La enseñanza implica un diálogo entre el profesor y el estudiante, con el fin de que este último se encause hacia la asimilación y la adopción de una serie de conocimientos y hacia el manejo de conceptos fundamentales. La comunicación provee un espacio para exteriorizar las dudas, incertidumbres, reflexiones o dilemas, que surgen en esta interacción.

El diálogo se rompe cuando no se le permite al estudiante participar activamente en el proceso, o bien, se le inhibe su potencial de generar conocimiento en conjunto con el profesor. El hilo conductor del diálogo debe ser el conocimiento y para su activación se requiere de un código común. Además, una buena comunicación necesita de ciertas reglas como respeto mutuo, honestidad, interés, deseo de superación, consistencia, etc. Por último, un buen diálogo obliga a tomar una posición tolerante ante las divergencias de opinión de los alumnos.

\section{RETOMANDO EL PROBLEMA INICIAL}

Según el problema inicial, la necesidad de contar con criterios que permitan valorar la efectividad de los materiales didácticos, es posible "rescatar", en un sentido no peyorativo, sino con una intención de adaptabilidad de criterios, algunas ideas de lo expuesto en la sección anterior para que sirva de base en la consecución del objetivo planteado.
En primer instancia, conviene aclarar que el propósito fundamental es encontrar el método adecuado para extraer el máximo rendimiento de la interacción estudiante-objeto, dentro de un proceso de aprendizaje.

En su trabajo, Los métodos de enseñanza en la universidad, el profesor Tito Quirós señala algunas pautas que serán tomadas como referencia en este trabajo:

i) "Los materiales deben posibilitar el acceso a la información de tal modo que faciliten la percepción de la realidad.

ii) Los materiales deben permitir a los estudiantes operar sobre ellos, cuanto más manipulables sean, más facilitan el trabajo del alumno.

iii) Los materiales deben ser concebidos para que sean aplicables a uno o más usos específicos por parte de los estudiantes y no sólo como elementos complementarios para la actividad del docente (...)" [4]

Otros requisitos para ser considerados son los siguientes:

iv) El diseño de un material o equipo didáctico debe obedecer a un objetivo claro, al mismo tiempo, deberá estar dirigido al estudian-te -el verdadero usuario- de forma tal que se adapte a sus características culturales y psicológicas.

v) La operación del equipo debe ser claramente explicada a través de manuales de uso, simbología, colores, formas, etc.

vi) En cuanto a la "interface" usuario/equipo, debe proporcionar un buen canal de comunicación de manera que permita la construcción de conocimiento; en otras palabras, los equipos deberían presentar la posibilidad de realizar cuestionamientos, como por ejemplo ¿qué pasa si....? , y de devolver la información al estudiante para que pueda ser descodificada. 
vii) Uno de los rasgos, que sería deseable encontrar en los equipos didácticos, es el en-foque de los mapas conceptuales, pues brinda al estudiante una buena estrategia para poder integrar el desarrollo del conocimiento dentro de su subjetividad.

Por último, la profesora Ma. Angélica Olivares[3] propone algunos conceptos para la evaluación de programas educativos de computación:

Comunicabilidad: Se refiere a la capacidad que tiene el dispositivo en cuestión para transmitir de manera clara, correcta, adecuada y significativa un mensaje educativo.

Acción: Consiste en evaluar si el objeto en estudio induce al sujeto a realizar acciones que posibiliten cambios en la estructura del conocimiento.

Efectividad: Se refiere a la capacidad de verificar los alcances del estudiante en su interacción con el equipo.

Parsimonia: Considera la sobriedad y moderación del objeto así como las acciones que son realizadas por el estudiante.

Por último se recomienda realizar una evaluación de los materiales didácticos, de ma-nera previa a su incorporación dentro del proceso de enseñanza y durante la misma con el fin de observar las reacciones y aprendizajes que provoca en los estudiantes.

\section{CONCLUSIONES Y RECOMENDACIONES}

Los contenidos de este artículo permiten concluir que la valoración de los equipos di-dácticos no se ha abordado con suficiente profundidad en la lit- eratura especializada, dado que se han presentado dificultades para localizar información específica sobre el tema; al respecto planteamos algunas recomendaciones:

1. Iniciar investigaciones relacionadas con las siguientes interrogantes: ¿Existe una diferencia entre los equipos didácticos y los equipos de experimentación?; ¿Deben los equipos didácticos llevar al estudiante a la experimentación formal?.

2. En futuras investigaciones, brindar una mayor clarificación de criterios, pues permitirá establecer bases y lineamientos para diseñar adecuadamente el equipo y el material didáctico empleado en los cursos de Ingeniería.

\section{BIBLIOGRAFÍA}

1. [1] Abarca M., Sonia. Psicología de la Educación. Centro de Investigación y Perfec-cionamiento para la Educación Técnica. (CIPET). Ministerio de Educación Pública. 1994.

2. [2] Murillo Ch., Fernando. Manual de Microenseñanza, Seminario "Técnicas Didácti-cas, para la Capacitación de Personal". Universidad de Costa Rica, Centro de Investi-gación y Capacitación en Administración Pública (CICAP). 1994.

3. [3] Olivares Z., María Angélica. Evaluación de Software educativo: Una Propuesta. Revista A la Carta, Noticias de Educación para América Latina. Número 7, febrero, 1994.

4. [4] Quirós R., Tito. Los Métodos de Enseñanza en la Universidad. Departamento de Docencia Universitaria, Universidad de Costa Rica, 1994. 